\title{
Dehiscencia y evisceración vaginal recidivante. A propósito de un caso
}

\author{
Gador Manrique Fuentes ${ }^{1}$, Rosario Redondo Aguilar ${ }^{1}$, Leonardo Mauro Aisa Denaroso ${ }^{1}$, \\ Luis Delgado Martínez ${ }^{1}$, Valois González Acosta ${ }^{1}$, Longinos Aceituno Velasco. ${ }^{1}$ \\ ${ }^{1}$ Unidad de Gestión Clínica de Obstetricia y Ginecología, Hospital La Inmaculada, Área de Gestión Sanitaria Norte de \\ Almería, Servicio Andaluz de Salud. Almería, España.
}

\section{RESUMEN}

La dehiscencia de la cúpula vaginal, con o sin evisceración, es una complicación rara de la cirugía ginecológica. El principal factor de riesgo es el antecedente de una histerectomía, a lo que se añaden otros factores desencadenantes, entre los que destaca la atrofia vaginal. El diagnóstico de evisceración es fácil, al comprobar salida de material intestinal a través de la vagina, siendo más dificultoso el diagnóstico de dehiscencia, ya que puede acontecer con síntomas banales. El tratamiento supone una urgencia quirúrgica al existir riesgo de lesión intestinal y peritonitis, pudiéndose realizar diferentes vías de abordaje teniendo en cuenta sobre todo la clínica de la paciente y tras comprobar el estado del contenido eviscerado. Presentamos el caso de una paciente con varios factores de riesgo, que presentó dehiscencia de la cúpula vaginal con evisceración de intestino de manera reiterada, y a la que se le practicó tratamiento quirúrgico por diferentes vías de abordaje así como tratamiento médico.

\section{PALABRAS CLAVE: Dehiscencia cúpula vaginal, evisceración intestinal, histerectomía previa, atrofia vaginal, urgencia quirúrgica}

\section{SUMMARY}

Dehiscence of the vaginal vault with or without evisceration is a rare complication after gynaecological surgery. The main risk factor is a previous hysterectomy, with the addition of other trigger conditions, the most important is vaginal atrophy. The diagnosis of evisceration is easy by confirmation the loosing of intestinal contents through the vagina. Diagnosis of dehiscence is more difficult because its symptoms could be more generic. The treatment is an emergency surgery as there is a risk of intestinal injury and peritonitis. There could be different surgical approaches taking into consideration the patient's clinic symptoms and after checking the state of eviscerated tissues. We expose the case of a patient with several risk factors, who presented dehiscence of the vaginal vault with recurrent bowel evisceration, and who we performed surgical treatment by different approaches and medical treatment.

KEY WORDS: Vaginal vault dehiscence, intestinal evisceration, prior hysterectomy, vaginal atrophy, surgical emergency 


\section{INTRODUCCIÓN}

La dehiscencia de la cúpula vaginal, con o sin evisceración, es una complicación rara de la cirugía ginecológica. La dehiscencia del manguito vaginal se define como la separación parcial o total de los bordes anterior y posterior de la cúpula vaginal, mientras que la evisceración se refiere al prolapso de contenido intraperitoneal (típicamente intestino delgado -íleon-) a través del defecto del manguito, siendo la localización del defecto más frecuente el fondo de saco vaginal posterior.

Se estima que la dehiscencia vaginal ocurre entre el 0,24 - 0,39\% de los casos tras una histerectomía, siendo esta intervención el principal factor de riesgo para su aparición. La evisceración se observa aproximadamente en el $35-67 \%$ de todas las dehiscencias de la cúpula vaginal (1) .

Además del antecedente de una histerectomía, se han descrito múltiples factores de riesgo relacionados con la dehiscencia vaginal, entre otros la realización del coito antes de la curación completa de la cúpula vaginal, la atrofia vaginal, y la presencia de prolapso de cúpula vaginal de larga evolución o de enterocele concomitante.

El cuadro de evisceración vaginal tiene un fácil diagnóstico tras comprobar la salida de material intestinal a través de la vagina, resultando más difícil diagnosticar una dehiscencia de vagina sin evisceración.

El tratamiento supone una urgencia quirúrgica. En casos de evisceración, es imprescindible comprobar la integridad intestinal, por lo que a la vía vaginal suele ser necesario añadir la vía abdominal o laparoscópica.

Presentamos el caso de una paciente con dehiscencia de la cúpula vaginal y evisceración de contenido intestinal, y posterior recidiva pese a tratamiento médico y quirúrgico en varias ocasiones.

\section{Caso clínico}

Paciente de 71 años, con los siguientes antecedentes personales: Diabetes Mellitus insulino dependiente tipo II, hipertensión arterial, obesidad, depresión, miocardiopatía hipertensiva, accidente cerebro vascular, ateromatosis carotídea, aneurisma interauricular con foramen oval permeable, dislipemia, poliartrosis, hernia discal, y antecedentes quirúrgicos de adenoma tubular colónico, tumor fibromixoide de muslo, adenoma tiroideo tóxico (hemitiroidectomía izquierda).

Como antecedentes gineco-obstétricos: menarquia a los 11 años, FO: 60666 (partos eutócicos), menopausia quirúrgica a los 45 años. En el año
1990, se realiza histerectomía total abdominal y doble anexectomía por útero miomatoso. En 2008, tras diagnóstico de cistocele de III grado sintomático, se realiza reparación, con colocación de malla vaginal anterior.

En 2010, la paciente acude a urgencias por salida de material intestinal a través de vagina, se diagnostica dehiscencia de cúpula vaginal con evisceración intestinal y se realiza intervención inmediata con sutura de cúpula vaginal por vía vaginal y laparoscopia exploradora con comprobación de integridad del intestino prolapsado. Al alta se indica tratamiento con estrógenos locales (crema de Estriol 0,1\%). En la revisión postquirúrgica, un mes después, se visualiza prolapso de la cúpula vaginal, indicándose colocación de pesario vaginal (no75).

A lo largo del año 2011 y 2012 acude a urgencias en varias ocasiones por caída de pesario y prolapso de cúpula vaginal, sobre todo en compartimento anterior. Se intenta reiteradamente colocar un pesario de mayor tamaño, siempre con caída del mismo. Tras intentar colocar pesario no 95 se visualiza vagina muy eritematosa e irritada, por lo que se consensúa con la paciente realizar plastia vaginal anterior, aconsejándose colposacropexia en caso de recurrir la patología. La plastia vaginal se realizó en noviembre de 2013. En mayo de 2014, en una revisión ginecológica, se visualiza prolapso total de cúpula vaginal, y lesión ulcerada en polo superior de cúpula vaginal. Se indica tratamiento con Blastoestimulina (centella asiática, neomicina) y se coloca nuevamente pesario vaginal, a la espera de realizarse una colposacropexia.

En julio de 2014 acude a urgencias por salida de material intestinal por vagina (Figura 1). Ante una recidiva de la evisceración vaginal, se indica intervención conjunta entre el Servicio de Ginecología y de Cirugía para realizar colposacropexia de manera urgente. La paciente se encuentra clínica y hemodinámicamente estable, y el hemograma tan solo muestra una ligera desviación a la izquierda en la serie leucocitaria, sin leucocitosis. Se indica cirugía por vía laparotómica ante la complejidad de la intervención por recidiva. Intraoperatoriamente no se visualizaron signos de isquemia o lesión intestinal y los bordes de la dehiscencia de cúpula vaginal no se observaron necrosados. En la intervención se realiza: reducción del material intestinal prolapsado, colporrafia con sutura de reabsorción lenta con puntos sueltos, tras desbridamiento de bordes a aproximar, colocación de malla en cúpula vaginal, colposacropexia, apendicectomía profiláctica, colocación de malla subperitoneal profiláctica por obesidad y colpocleisis (Figura 2). 


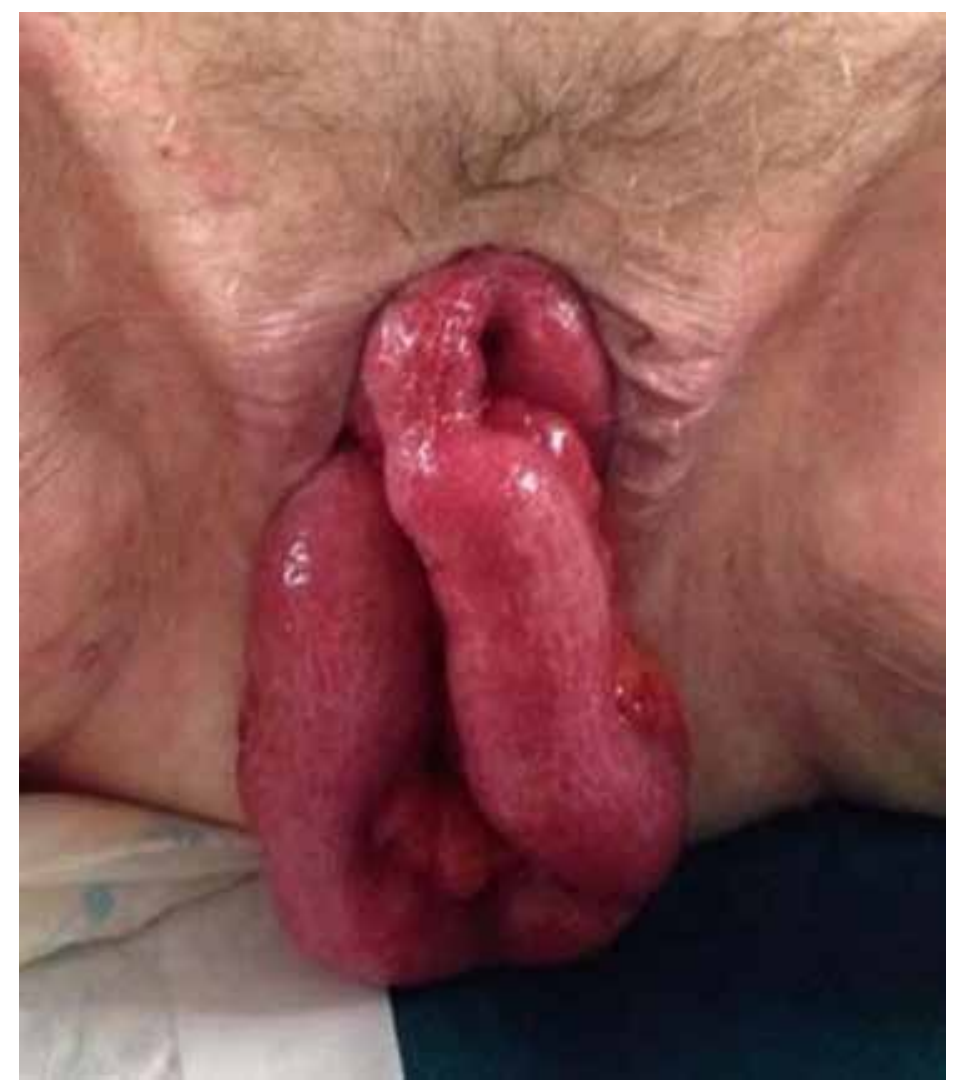

Figura 1. Caso clínico: Evisceración vaginal.

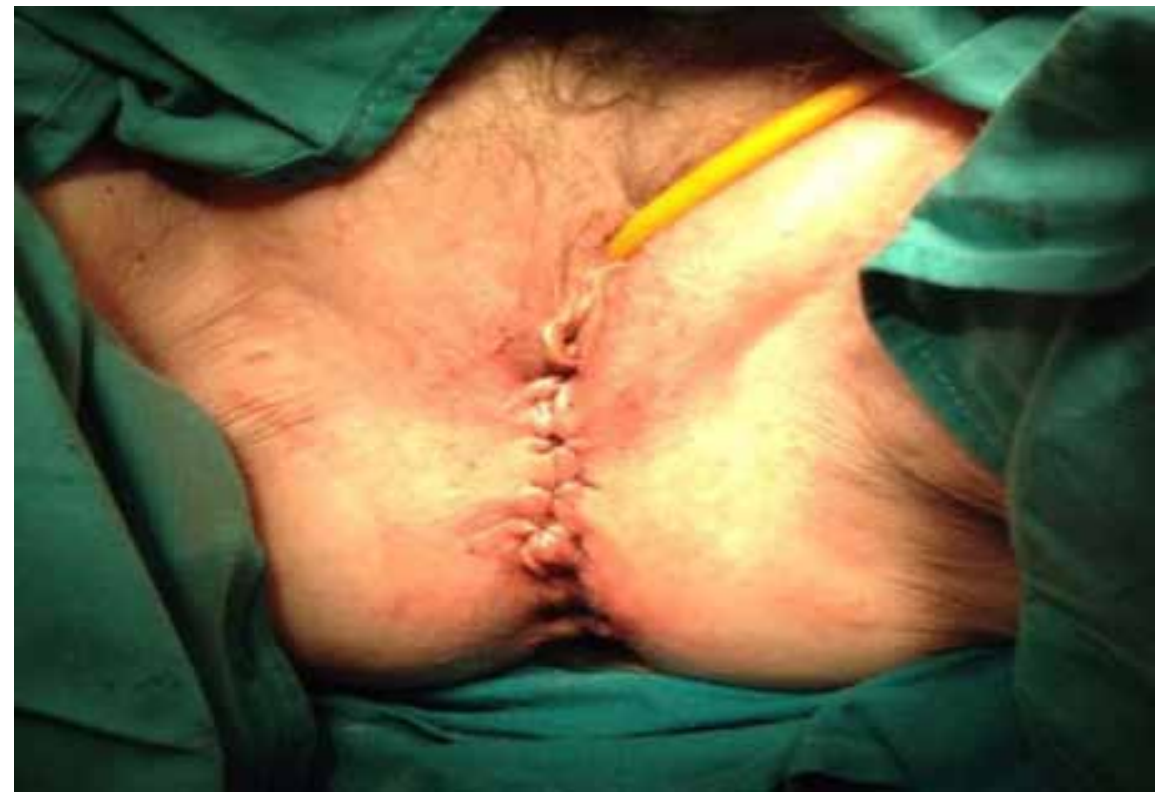

Figura 2. Caso clínico: Colpocleisis. 
Durante el posoperatorio la paciente recibe tratamiento antibiótico de amplio espectro con Clindamicina y Gentamicina, con el que continúa durante una semana tras obtener el resultado del cultivo abdominal realizado intraoperatoriamente (cultivo positivo a Staphylococcus Aureus y a Serratia Marcescens). La paciente es dada de alta al tercer día de la intervención, tras buena evolución.

En la revisión postquirúrgica (septiembre de 2014) la paciente se encuentra asintomática, la cleisis vaginal y la herida de la laparotomía se encuentran en buen estado.

En noviembre de 2014 acude a urgencias por sensación de bulto en genitales externos, diagnosticándose nuevamente cistocele, además de atrofia vaginal. Se indica tratamiento con crema de Promestrieno y Blastoestimulina y se coloca pesario vaginal. En la última revisión, la mucosa vaginal se encuentra en buen estado, con buena tolerancia del pesario no 80 .

\section{DISCUSIÓN}

La dehiscencia de la cúpula vaginal, con o sin evisceración, es una complicación rara de la cirugía ginecológica. En una amplia revisión realizada por Kowalski y cols (1), de 1901 a 1996, en la bibliografía mundial sólo se habían informado 60 casos.

El principal factor de riesgo para la aparición de una dehiscencia vaginal es el antecedente de una histerectomía, a lo que se añaden otros factores desencadenantes. En el caso de las pacientes premenopausicas, el principal factor desencadenante es la interrupción de la cúpula vaginal secundario a un coito antes de completar la curación después de la cirugía; y para las mujeres posmenopáusicas, el prolapso de la cúpula vaginal de larga evolución y la aparición de enterocele son los factores sobreañadidos más importantes, a lo que se suma el estado de atrofia genital por hipoestrogenismo (2).

El tipo de histerectomía vaginal ha sido evaluada en varios estudios, aunque debido a la baja incidencia de la entidad, no hay conclusiones definitivas sobre qué vía se relaciona en mayor medida con la evisceración vaginal.

Ramírez y Klemer (3), revisaron la incidencia de herniación vaginal y encontraron que el $62 \%$ de las mujeres tenían antecedentes de histerectomía por vía vaginal, 32\% por histerectomía por laparotomía, y $5 \%$ de histerectomía laparoscópica.

El potencial riesgo de dehiscencia del manguito vaginal con la cirugía laparocópica y robótica, se justifica por los siguientes factores: uso de fuentes de energía para colpotomía y magnificación del campo quirúrgico que puede resultar en suturas en campos más superficiales y pobre integridad del nudo (4).

Hur y cols (5), encontraron un mayor riesgo de desarrollar la evisceración después de una histe- rectomía laparoscópica en comparación con las vías vaginal o abdominal, describieron una incidencia de dehiscencia del $0,26 \%$ si el abordaje de la histerectomía previa era abdominal, del 0,25\% si era vaginal y hasta del $0,79 \%$ si la vía había sido laparoscópica (diferencias estadísticamente significativas). laco y cols (6), también señalaron una mayor incidencia de evisceración tras el abordaje laparoscópico.

En el caso que nos ocupa, la paciente cuenta con varios factores de riesgo para la evisceración vaginal:

- Fue sometida a una histerectomía abdominal, tras lo cual tuvo prolapso genital (cistocele) de larga evolución, que precisó reparación quirúrgica con colocación de malla vaginal e indicación de pesario vaginal por recidiva del prolapso.

- La histerectomía realizada se acompañó de doble anexectomía, lo que ocasionó una menopausia quirúrgica a los 45 años. La gran atrofia vaginal que presenta la paciente en las sucesivas revisiones, debido a ese estado de hipoestrogenismo, se ve agravada por la exposición de la mucosa vaginal por el prolapso y el efecto inflamatorio sobre la misma tras la colocación del pesario vaginal. Tras la primera intervención fue correcta la indicación de estrógenos locales.

El diagnóstico de la dehiscencia del manguito vaginal con o sin evisceración, es clínico. La clínica de la evisceración es patognomónica: salida de material intestinal a través de la vagina. Sin embargo, la herniación sin evisceración puede acontecer con síntomas muy banales, tales como sangrado vaginal escaso o flujo vaginal, dolor abdominal leve o sensación de peso en genitales, debiéndose sospechar el cuadro ante pacientes con factores de riesgo. Ante estos síntomas se debe realizar un examen físico urgente, incluida una evaluación de los signos vitales, palpación abdominal para detectar signos de peritonitis, y un cuidadoso examen vaginal. Si no se visualiza contenido peritoneal en el introito, se introducirá un espéculo con cuidado para descartar la presencia de dehiscencia, hematoma, celulitis o absceso. A ello se sigue la palpación manual del manguito para confirmar la integridad. Si el contenido peritoneal es visible a través del introito, se ha de envolver en una toalla húmeda en espera de traslado a quirófano.

Los estudios de laboratorio recomendados incluyen: hemograma y pruebas de coagulación. Una leucocitosis significativa sugiere una infección intestinal o isquemia intraabdominal que impide un abordaje único transvaginal seguro. Las pruebas de imagen adicionales como el TAC solo estarían indicadas ante la sospecha clínica de absceso, hematoma o lesión intestinal.

En nuestro caso, el diagnóstico resultó fácil en ambas ocasiones, ya que la paciente acude a urgencias tras dehiscencia y prolapso de intestino 
delgado a través de la vagina. Tanto en la primera ocasión como en la recidiva posterior, la clínica consistió en dolor abdominal leve, sin signos de irritación peritoneal a la exploración ni tras la realización de la analítica, y sin evidencia de lesión o isquemia del intestino delgado prolapsado tras comprobación laparoscópica en la primera intervención, y laparotómica en la segunda.

La evisceración vaginal es una emergencia médica que requiere una intervención quirúrgica inmediata por el potencial riesgo de lesión intestinal y perinonitis, motivo por el cual se recomienda iniciar antibioterapia IV de amplio espectro desde el momento del ingreso (7). El contenido eviscerado puede lesionarse por un traumatismo directo en el segmento expuesto o a través de la isquemia secundaria a encarcelamientos con posterior estrangulación.

La intervención quirúrgica consta de varias fases: 1) reducción del contenido abdominal eviscerado, 2) evaluación de la viabilidad del intestino y resección del isquémico, 3) extirpación del tejido vaginal desvitalizado, y lavado de las superficies contaminadas, 4) reparación del defecto vaginal, mediante puntos con material no-absorbible o colocación de una malla no absorbible de polipropileno. En la literatura se han descrito abordajes vaginal y abdominal (laparotómico y laparoscópico) puros, o una combinación de ambos.

Históricamente, los libros de texto abogaban fuertemente por la laparotomía media inmediata y no se aceptaba la reparación transvaginal. Sin embargo, más recientemente, se han descrito resultados exitosos utilizando un abordaje vaginal o laparoscópico, en asociación con una creciente prevalencia en la cirugía mínimamente invasiva (8).

Actualmente, parece razonable que el abordaje quirúrgico sea dictado en función del estado general de la paciente y del estado del intestino eviscerado. Así, el cierre transvaginal de la dehiscencia vaginal se reserva para los pacientes médicamente estables y que no tienen evidencia clínica de peritonitis o lesión intestinal. En aquellos casos en los que el segmento eviscerado apareciese oscuro o traumatizado, o si la cínica o la analítica sugiriesen isquemia intestinal, peritonitis, hematoma o absceso, se precisaría una evaluación intraabdominal, utilizando laparotomía o laparoscopia, dependiendo de la habilidad quirúrgica individual y del estado general del paciente.

En el caso que nos ocupa, en las dos ocasiones en las que se produjo la evisceración, el intestino delgado no sufrió daño alguno, y permitió la reparación vía vaginal, eso sí, con confirmación de la integridad intestinal, la primera vez a través de laparoscopia, y tras la recidiva mediante laparotomía, dada la indicación de colposacropexia y preferencia de cirujanos.
La recurrencia de una evisceración vaginal es un fenómeno tan raro, que no existe suficiente evidencia que recomiende una técnica sobre otra, con vistas a evitarla.

Tras la intervención, los pacientes permanecen ingresados hasta que hayan demostrado retorno de la función intestinal y no tengan evidencia clínica ni analítica de enfermedad sistémica. Es importante mantenerse alerta ante la posibilidad de isquemia mesentérica.

Se aconseja mantener relaciones sexuales sólo después de demostrar la curación completa del manguito. En las mujeres posmenopáusicas, se puede utilizar crema vaginal de estrógenos para ayudar en la cicatrización de la herida (9).

\section{CONCLUSIONES}

La dehiscencia de la cúpula vaginal, con o sin evisceración vaginal, es una entidad rara, cuyo principal factor de riesgo es el antecedente de histerectomía previa. Cuando se diagnostica, se trata de una urgencia quirúrgica, por el potencial riesgo de lesión intestinal y perinonitis. No está claro cual es el mejor abordaje ni la mejor técnica quirúrgica, sobre todo con vistas a disminuir el riesgo de recidiva, pero sí se sabe que la vía de abordaje debe ser dictada por el estado general del paciente y el estado del intestino prolapsado. Se acepta el abordaje únicamente por vía vaginal solo tras haber descartado una posible lesión intestinal o peritonitis, pudiéndose de esta forma evitar la morbilidad asociada al abordaje abdominal. La vía abdominal (laparotómica o laparoscópica) es la de elección cuando existe evidencia o sospecha de traumatismo o isquemia intestinal. La atrofia vaginal es otro factor de riesgo a considerar en pacientes postmenopausicas, por lo que el tratamiento con estrógenos locales puede ser útil tras la reparación del defecto vaginal, sobre todo en aquellas pacientes con hipoestrogenismo de larga evolución, y en las que se asocian otros factores de riesgo.

\section{REFERENCIAS}

1. Kowalski LD, Seski JC, Timmins PF, Kanbour Al, Kunschner AJ. Vaginal evisceration: presentation and management in postmenopausal women. J Am Coll Surg 1996;183:225-9.

2. Woo KC, Linden JA, Lowenstein RA, et al. Subtle vaginal evisceration resulting in small bower evisceration: a case report. Am J Emerg Med 2012;43;e125e128.

3. Ramírez PT, Klemer DP. Vaginal evisceration after hysterectomy: a literature review. Obstet Gynecol Surv 2002;57:462-7.

4. Fanning J, Kesterson J, Davies M, Green J, Penezic $\mathrm{L}$, Vargas $\mathrm{R}$, et al. Effects of electrosurgery and 
vaginal closure technique on postoperative vaginal cuff dehiscence. JSLS 2013;17:414-7.

5. Hur HC, Guido RS, Mansuria SM, Hacker MR, Sanfilippo JS, Lee TT. Incidence and patient characteristics of vaginal cuff dehiscence after different modes of hysterectomies. J Minim Invasive Gynecol 2007;14:3117.

6. Iaco PD, Ceccaroni M, Alboni C, Roset B, Sansovini $M$, D'Alessandro $L$, et al. Transvaginal evisceration after hysterectomy: is vaginal cuff closure associated with a reduced risk? Eur J Obstet Gynecol Reprod Biol 2006;125:134-8.
7. Matthews CA, Kenton K. Treatment of vaginal cuff evisceration. Obstet Gynecol 2014;124:705-8.

8. Uccella S, Ceccaroni M, Cromi A, Malzoni M, Berretta $R$, De laco $P$, et al. Vaginal cuff dehiscence in a series of 12,398 hysterectomies: effect of different types of colpotomy and vaginal closure. Obstet Gynecol 2012;120:516-23.

9. Vesna A, Neli B. Benefit and safety of 28-day transdermal estrogen regimen during vaginal hysterectomy (a controlled trial). Maturitas 2006;53:282-98. 